\title{
Stepwise maturation of the peptidyl transferase region of human mitoribosomes
}

\section{Journal Article}

\section{Author(s):}

Lenarcic, Tea (D); Jaskolowski, Mateusz; Leibundgut, Marc; Scaiola, Alain (iD); Schönhut, Tanja; Saurer, Martin; Lee, Richard G.; Rackham, Oliver; Filipovska, Aleksandra; Ban, Nenad

Publication date:

2021-12-01

\section{Permanent link:}

https://doi.org/10.3929/ethz-b-000491295

\section{Rights / license:}

Creative Commons Attribution 4.0 International

\section{Originally published in:}

Nature Communications 12(1), https://doi.org/10.1038/s41467-021-23811-8

\section{Funding acknowledgement:}

182341 - Structural studies of eukaryotic ribosomal complexes involved in regulation of translation and in mitochondrial protein synthesis (SNF)

182880 - NCCR RNA\&Disease (51NF40-182880): Flexibility Grant (SNF) 
ARTICLE

\section{Stepwise maturation of the peptidyl transferase region of human mitoribosomes}

Tea Lenarčič ${ }^{1,6}$, Mateusz Jaskolowski ${ }^{1,6}$, Marc Leibundgut', Alain Scaiola (1) 1, Tanja Schönhut ${ }^{1}$, Martin Saurer ${ }^{1}$, Richard G. Lee ${ }^{2,3}$, Oliver Rackham 2,3,4,5, Aleksandra Filipovska (i) ${ }^{2,3,5} \&$ Nenad Ban (1) ${ }^{1 凶}$

Mitochondrial ribosomes are specialized for the synthesis of membrane proteins responsible for oxidative phosphorylation. Mammalian mitoribosomes have diverged considerably from the ancestral bacterial ribosomes and feature dramatically reduced ribosomal RNAs. The structural basis of the mammalian mitochondrial ribosome assembly is currently not well understood. Here we present eight distinct assembly intermediates of the human large mitoribosomal subunit involving seven assembly factors. We discover that the NSUN4MTERF4 dimer plays a critical role in the process by stabilizing the 16S rRNA in a conformation that exposes the functionally important regions of rRNA for modification by the MRM2 methyltransferase and quality control interactions with the conserved mitochondrial GTPase MTG2 that contacts the sarcin-ricin loop and the immature active site. The successive action of these factors leads to the formation of the peptidyl transferase active site of the mitoribosome and the folding of the surrounding rRNA regions responsible for interactions with tRNAs and the small ribosomal subunit.

\footnotetext{
${ }^{1}$ Department of Biology, Institute of Molecular Biology and Biophysics, ETH Zurich, Zurich, Switzerland. ${ }^{2}$ Harry Perkins Institute of Medical Research, QEII Medical Centre, University of Western Australia, Nedlands, WA, Australia. ${ }^{3}$ ARC Centre of Excellence in Synthetic Biology, QEII Medical Centre, University of Western Australia, Nedlands, WA, Australia. ${ }^{4}$ Curtin Health Innovation Research Institute and Curtin Medical School, Curtin University, Bentley, WA, Australia. ${ }^{5}$ Telethon Kids Institute, Northern Entrance, Perth Children's Hospital, Nedlands, WA, Australia. ${ }^{6}$ These authors contributed equally: Tea Lenarčič, Mateusz Jaskolowski. ${ }^{凶}$ email: ban@mol.biol.ethz.ch
} 
$\mathrm{H}$ uman mitochondrial ribosomes (mitoribosomes) are responsible for the synthesis of 13 oxidative phosphorylation (OXPHOS) proteins, encoded by the mitochondrial genome $e^{1,2}$. Due to their unusual architectural features ${ }^{1-3}$ and the requirement to coordinate mitochondrial ribosomal RNA (rRNA) synthesis with the import of all ribosomal proteins, their assembly is anticipated to involve mitochondrial-specific pathways and participation of both conserved and mitochondrialspecific maturation factors, such as GTPases and methyltransferases ${ }^{1}$. Production of functional mitoribosomes is critical since defects in the mitochondrial translation machinery lead to a range of severe human diseases ${ }^{4}$.

Structural analyses of the yeast ${ }^{5}$ and prokaryotic cytoplasmic large subunits ${ }^{6,7}$ as well as kinetoplastid mitoribosomal large subunit (mt-LSU) assembly intermediates ${ }^{8-10}$, combined with the biochemical studies of yeast and human mitoribosomal assembly ${ }^{11,12}$, showed that the solvent exposed side of the mtLSU matures early, whereas the conserved functional sites of the large subunit, such as the peptidyl transferase center (PTC), the GTPases-associated center (GAC) and the polypeptide exit tunnel, assemble last. At the final stages of the maturation, assembly factors bind to these functional regions to facilitate their maturation and prevent premature translation ${ }^{13}$. Although human mitoribosome assembly has been extensively investigated using a combination of biochemical and high-throughput approaches ${ }^{12,14-16}$, structural understanding of this process is currently limited to a late assembly intermediate where a MALSU1-L0R8F8-mt-ACP module is bound ${ }^{17}$.

To better understand the structural basis of human mitoribosome maturation, we isolated mt-LSU assembly intermediates and investigated their composition and structures using cryoelectron microscopy (cryo-EM). We determined structures of eight distinct states of the human mt-LSU where a total of seven assembly factors (MTERF4, NSUN4, MRM2, MTG2, MALSU1, L0R8F8, and mt-ACP) were bound in different combinations. Our results reveal the conformational changes that allow successive modification and maturation of the functionally important regions of rRNA. The structural data supported by biochemical evidence provide an explanation for the role of the essential NSUN4-MTERF4 heterodimer in the process and specifically in the maturation of the functionally important tRNAinteracting P loop through interplay with the conserved GTPase MTG2 (GTPBP5). The obtained results allow us to propose a stepwise maturation pathway of the functionally important regions in the human mitochondrial large ribosomal subunit (mtLSU).

a

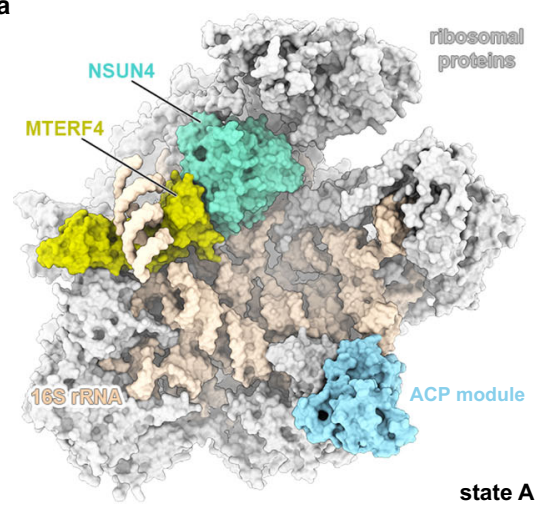

b

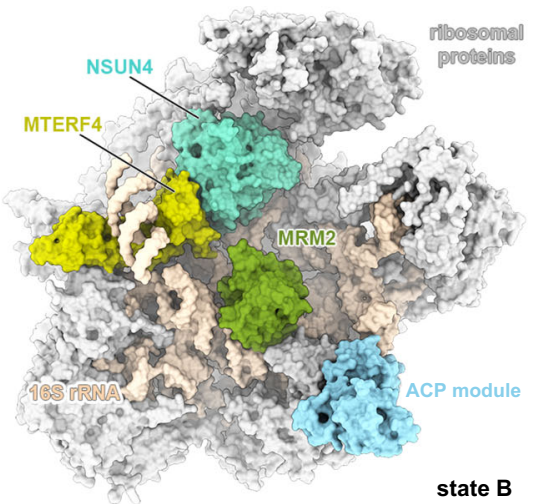

\section{Results}

Structures of assembly intermediates of the human mitoribosomal large subunit. To purify assembly intermediates of the human mt-LSU, we transfected human embryonic kidney cells with a tagged mitochondrial GTPase 1 (MTG1) (Supplementary Fig. 1), which is a homolog of bacterial $\operatorname{RbgA}^{18,19}$ and is essential for the production of functional mitoribosomes due to its involvement in the late stages of mt-LSU assembly prior to monosome formation ${ }^{20,21}$. The affinity-purified sample was investigated using single-particle cryo-EM to reveal several mt-LSU-like structures containing the entire set of ribosomal proteins. Furthermore, density for several additional proteins was observed at the intersubunit side bound to the rRNA in an immature conformation. Using focused classification around those additional features, we were able to obtain reconstructions of eight distinct cryo-EM classes (overall resolution range between 2.9 and $3.5 \AA$ ) corresponding to assembly intermediate states of the mt-LSU (Supplementary Figs. 2 and 3). Two of the classes, states A0 and $\mathrm{D}$, resemble the structures of the assembly intermediates described previously, where a MALSU1-L0R8F8-mt-ACP module is bound at the intersubunit face, whereas the rRNA is either disordered or in a nearly mature state, respectively ${ }^{17}$. Other classes correspond to novel assembly intermediates (Supplementary Fig. 2). We characterized in detail three of these intermediates, resolved to 2.9, 3.1, and $3.1 \AA$, and referred to them as states A, B, and C, respectively (Fig. 1 and Supplementary Table 1). They represent the key steps in the late stages of mt-LSU maturation, during which functionally important regions of the rRNA progressively mature. The remaining classes (states $\mathrm{C} 0, \mathrm{D}^{\prime}$, and $\mathrm{D0}^{\prime \prime}$ ) correspond to structurally related, but less complete states that occur between the described intermediates.

NSUN4-MTERF4 dimer stabilizes the rRNA in a conformation that exposes the active site region. In addition to the previously characterized MALSU1-L0R8F8-mt-ACP module ${ }^{17}$, state A contains at the intersubunit side, below the central protuberance (CP), a dimer of NSUN4 and MTERF4, which were both previously identified as mitoribosomal assembly factors ${ }^{22,23}$ and their structures determined by x-ray crystallography 24,25 (Fig. 1a). Although tagged MTG1 was used for affinity purification of ribosomal assembly intermediates, the factor was not sufficiently ordered on the mt-LSU to be structurally interpreted in any of the observed states. The NSUN4-MTERF4 dimer forms extensive interactions with the rRNA and ribosomal proteins by contacting rRNA helices H66, H75, H81, H87, and H93, and keeps the Ctermini of $\mathrm{uL} 2 \mathrm{~m}$ and $\mathrm{mL} 48$ in an immature conformation

Fig. 1 Structures of the key human mitoribosomal large subunit assembly intermediates. The molecular structures of states $A$ (a), $B$ (b), and $C$ (c) are shown in surface representation from the intersubunit side. Ribosomal proteins are depicted in light gray, 16S rRNA in beige, and assembly factors NSUN4, MTERF4, MRM2, MTG2, and the MALSU1-LOR8F8-mt-ACP (ACP) module in teal, light green, green, red, and blue, respectively. 
a

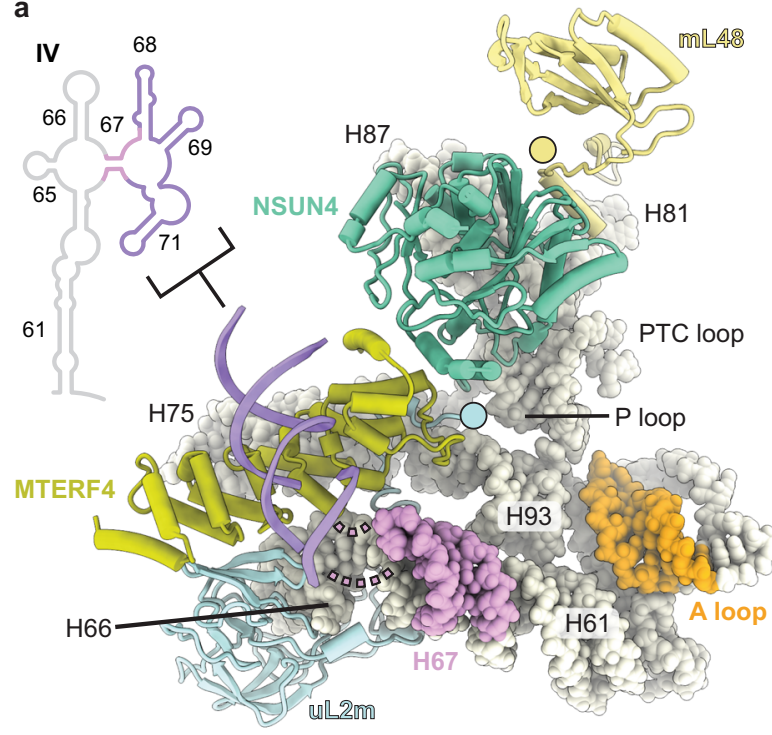

b

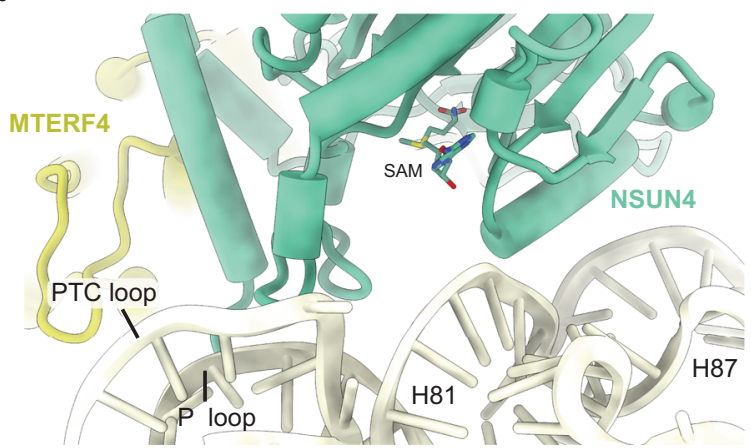

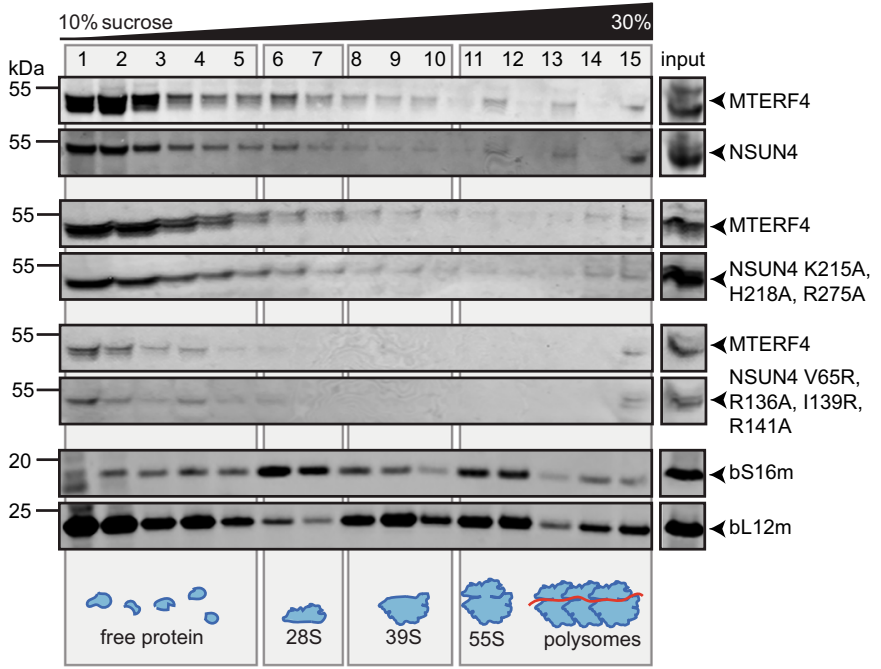

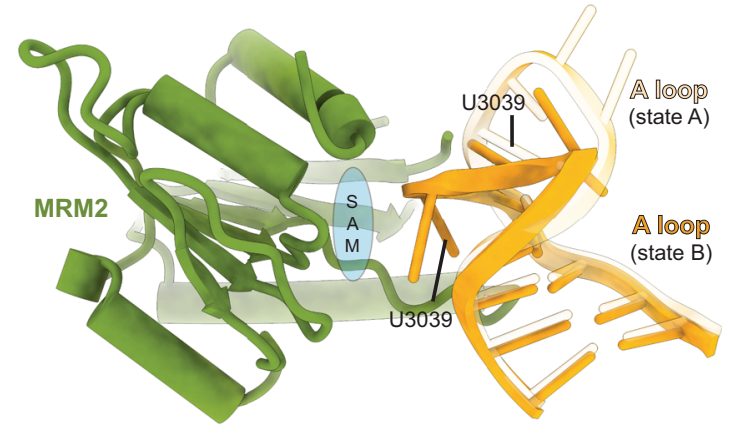

Fig. 2 Interactions of NSUN4 and MTERF4 with the immature human mitoribosomal large subunit. a The NSUN4-MTERF4 heterodimer (teal and light green, respectively) in state A forms a number of contacts with the mitochondrial large subunit via rRNA (beige spheres) and ribosomal proteins, resulting in rearrangements of the $\mathrm{mL} 48$ (yellow) and $\mathrm{uL} 2 \mathrm{~m}$ (light blue) C-termini (highlighted as circles), as well as stabilization of the $\mathrm{H} 68-\mathrm{H} 71$ region (violet) of the $16 \mathrm{~S}$ rRNA domain IV. The connection from helix $\mathrm{H} 67$ (pink) to H68-H71 is shown as dashed lines. The A loop, which is modified by MRM2 in state B, is colored orange. $\mathbf{b}$ The NSUN4 (teal) active site with bound S-adenosyl-methionine (SAM) cofactor in state A is shown together with nearby rRNA helices H81, H87, P loop, and PTC loop (beige cartoon). MTERF4 is also shown for orientation (light green). c Effects of mutations in NSUN4 on its association with mitoribosomal fractions were analyzed by immunoblotting. A continuous 10-30\% sucrose gradient was used to separate mitochondrial lysates from HEK 293T cells expressing MTERF4 and either the wild-type or a mutated version of NSUN4 to determine their distribution and co-migration with mitochondrial ribosomal fractions. The small and large ribosomal subunit and polysomes in mitochondria isolated from wild-type cells were followed by immunoblotting for mitochondrial ribosomal protein markers of the small (bS16m) and large (bL12m) ribosomal subunits. The input mitochondrial lysate was used as a positive control. The blots are representative of results obtained with at least three independent biological experiments. Source data are provided as a Source Data file. d Rearrangement of the A loop (light orange: state A; orange: state B) upon the MRM2 methylation event (state B). The likely position of MRM2 SAM cofactor, which is not visible in our structure, is schematically shown in blue.

(Fig. 2a). Interestingly, NSUN4 is an RNA $\mathrm{m}^{5} \mathrm{C}$ methyltransferase that has been implicated in the assembly of both small and large mitoribosomal subunits, however, biochemical data suggested that in vivo it only modifies the rRNA of the small subunit ${ }^{23}$. Consistently, no RNA substrate was found in the active site of NSUN4 where a clearly visible S-adenosyl-methionine (SAM) cofactor was bound (Fig. $2 \mathrm{~b}$ and Supplementary Fig. 4). Since NSUN4 lacks an RNA substrate recognition domain that is present in bacterial homologs ${ }^{22}$, it was proposed that it relies on interactions with MTERF4 to be targeted to the mt-LSU to regulate ribosome maturation ${ }^{22,23}$, as we now observe in the mtLSU-bound state.

The MTERF4 protein folds into a bent $\alpha$-solenoid that binds with its convex region to the surface of the immature subunit and exposes its positively charged concave region towards the outside, where we observe a segment of double-helical RNA bound (Supplementary Fig. 5). Although we could not assign the identity of RNA nucleotides in this region, continuous density can be traced from rRNA helix H67, suggesting that the additional density belongs to the H68-H71 region of the rRNA (Fig. 2a and Supplementary Fig. 5). In the mature mt-LSU these helices form the front of the peptidyl transferase active site cleft. The interactions with MTERF4 expose the immature rRNA regions corresponding to the PTC loop that forms the active site of the ribosome, as well as the A (H92) and $\mathrm{P}$ (H80) loops responsible for binding the acceptor end of tRNAs and positioning substrates for the peptidyl transferase reaction in the active site of the mature mt-LSU.

To better understand the role of both MTERF4 and NSUN4 in the context of the mt-LSU assembly, we designed a series of mutants based on our structural results with deletions in key interaction regions. The mutant proteins were then investigated with respect to their ability to form dimers and associate with the mitoribosome using continuous sucrose gradients in cells 
expressing a FLAG-tagged MTERF4 and HA-tagged NSUN4. Wild-type MTERF4 and NSUN4 associate with each other and co-migrate with the large subunit of the mitoribosome, confirming previous findings ${ }^{22-25}$. Two different mutants were designed to disrupt NSUN4 interactions with the 16S rRNA or MTERF4. The first NSUN4 mutant, bearing a triple mutation K215A, H218A and R275A located at the interface between the rRNA and NSUN4 (Supplementary Fig. 5), was designed to test the contribution of NSUN4 to binding of the complex to the $16 \mathrm{~S}$ rRNA of the immature mt-LSU. This mutation neither reduced the association of NSUN4 with MTERF4 nor their co-migration with the large subunit (Fig. 2c), indicating that MTERF4 plays a predominant role in delivering the complex to the immature subunit. A second NSUN4 mutant carrying a quadruple mutation of residues V65R, R136A, I139R, and R141A (Supplementary Fig. 5), designed to break the dimer between NSUN4 and MTERF4, completely abolished their interaction as shown previously ${ }^{25}$, and also prevented binding of either of the two proteins to the large subunit (Fig. 2c). We conclude that formation of a stable NSUN4-MTERF4 heterodimer is critical for their function in mitoribosome assembly.

MRM2 methylates the U3039 in the A loop before it adopts mature conformation. While state A described above contains only the MALSU1-L0R8F8-mt-ACP module and NSUN4-MTERF4 dimer, in state B we additionally observe the MRM2 methyltransferase bound to the rRNA in an optimal position for methylation of its target nucleotide U3039 within the A loop of the $16 \mathrm{~S}$ rRNA ${ }^{26,27}$ (Fig. 1b). The A loop is repositioned such that the 2'-O-ribose of nucleotide U3039 faces the active site of MRM2, although we do not observe density for a SAM cofactor (Fig. 2d). The binding site for the MRM2 methyltransferase, as observed in state B, is occupied by helix H71 in the mature mtLSU (Supplementary Fig. 6). This implies a temporal order of maturation events where U3039 methylation must take place before helix H71 assumes its mature conformation.

MTG2 and NSUN4 interact with the P loop in a tweezer-like manner. In state $\mathrm{C}$ (Fig. 1c), MRM2 is replaced by MTG2, a GTPase conserved from bacteria to eukaryotes. The bacterial homolog (ObgE) has been recently visualized on the native assembly intermediates of the bacterial large subunit ${ }^{7}$. MTG2 was proposed to play a key role in the human mt-LSU assembly as a final quality control checkpoint protein ${ }^{28}$. Furthermore, biochemical experiments showed that it interacts with the mt-LSU at the same time as MRM2, MTERF4, MTG1, and MALSU1 ${ }^{28}$. Our structure reveals that, in the presence of NSUN4, MTERF4, and the MALSU1-L0R8F8-mt-ACP module, MTG2 binds to the mtLSU in a position, from which it can simultaneously check two key regions of the large ribosomal subunit: the GAC that plays a key role in stimulating GTP hydrolysis of translational GTPases and the catalytic PTC (Fig. 3a).

On the side of the GAC, the conserved Ras-like G domain of MTG2 interacts with sarcin-ricin loop (SRL) H95 and ribosomal protein $\mathrm{uL11m}$ (Fig. 3b). The G domain is oriented such that the active site, including the only partially resolved G2/Switch I and G3/Switch II loops involved in GTP binding and hydrolysis, faces towards the SRL and $\mathrm{uL} 11 \mathrm{~m}$. Such positioning suggests that the G domain of MTG2 would be able to monitor the correct conformation of the GAC region in the final stages of mt-LSU maturation.

On the other side, the N-terminal Obg domain of MTG2 stabilizes the mature conformation of the PTC-forming helices H81, H89, H90 as well as the A and PTC loops and indirectly $\mathrm{H} 71$, which are in immature conformations in states $\mathrm{A}$ and B (Supplementary Fig. 7). The most prominent rRNA rearrangement involves the PTC loop, which in state A surprisingly forms a helix with the $\mathrm{P}$ loop of the 16S rRNA (Fig. 3c). As the PTC loop matures in state C, it withdraws from the $\mathrm{P}$ loop, which is now held in immature conformation by contacts with assembly factors MTG2 and NSUN4 (Fig. 3d). These interactions encompass the Obg domain of MTG2 and the N-terminal tail of NSUN4, which is disordered in the absence of MTG2. The two factors grip the immature P loop from two sides in a tweezer-like manner involving aromatic residues Tyr27 and Trp31 of NSUN4 that stack with the P loop nucleotides G2814 and A2817, whereas MTG2 contributes Phe92 to interact with the P loop nucleotide G2816 (Fig. 3d). While deletion of residues 26-36 at the N-terminal region of NSUN4 did not reduce the association of NSUN4 with MTERF4 or mt-LSU, it reduced the levels of mature mitoribosomes in a dominant-negative manner (Fig. 3e) compared to the wild-type control (Fig. 2c). Taken together, our structural and biochemical results reveal a direct contribution of the NSUN4 N-terminal tail to the maturation of the mt-LSU.

The above-mentioned $\mathrm{P}$ loop is the sole element of the $16 \mathrm{~S}$ rRNA domain $\mathrm{V}$ that remains immature in state $\mathrm{C}$ and, together with helices $\mathrm{H} 68$ and $\mathrm{H} 69$ of domain IV that are still bound to MTERF4, the only area in the mt-LSU rRNA that still needs to mature (Supplementary Fig. 7). Interestingly, besides keeping the P loop in a distinct immature conformation, MTG2 also samples the entrance to the nascent polypeptide tunnel with one of its Obg domain loops (Fig. 3f). At exit side of the ribosomal tunnel, we observe that mitoribosomal protein mL45 inserts its $\mathrm{N}$ terminal tail into the tunnel, as observed for the non-translating mature mitoribosomes ${ }^{29,30}$, and reaches almost to the Obg domain of MTG2. Consequently, the two proteins sample virtually the entire length of the exit tunnel and may play a role in facilitating proper folding of proteins and rRNA elements forming the mitoribosomal nascent polypetide tunnel during $\mathrm{mt}$ LSU maturation.

Stepwise maturation of the human mitochondrial large subunit. Visualization of eight distinct assembly intermediates of the human mt-LSU that reveal the interdependence of assembly factors and the role of the rRNA in the process allows us to propose a model for stepwise maturation of the ribosomal active site (Fig. 4 and Supplementary Fig. 8). Binding of the NSUN4-MTERF4 dimer to the immature, but compositionally complete mt-LSU, sequesters the flexibly disposed H68-H71 region to expose the active site and allow access of factors that modify the rRNA and check its conformation. This conformation is recognized by the methyltransferase MRM2 that modifies nucleotide U3039 in the A loop of the rRNA. After the methylation of U3039, MRM2 dissociates and the quality checkpoint GTPase MTG2 binds to the P loop in an NSUN4-dependent manner to facilitate maturation of the PTC and to check the functionality of the GAC of the ribosome. Once the rRNA assumes its native or nearly native conformation, the assembly factors dissociate, and a mature, translationally competent mtLSU is formed.

Our data provide the basis for understanding the late stages of human mt-LSU maturation at a structural level and reveal the key role of the NSUN4 and MTERF4 to induce a conformation of the subunit ready for subsequent modification and maturation steps. The described assembly intermediates show maturation and/or proof-reading of all functionally important regions of the large subunit. These results complement the discovery of a remarkably complex mitoribosomal assembly machinery in trypanosomal mitochondria ${ }^{8-10,31}$ and suggest that the divergent mitochondrial 
a

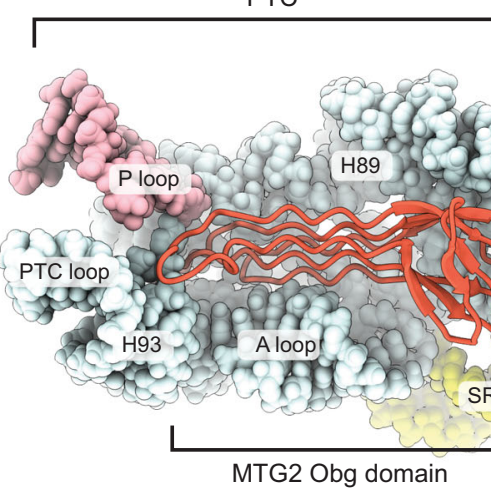

C

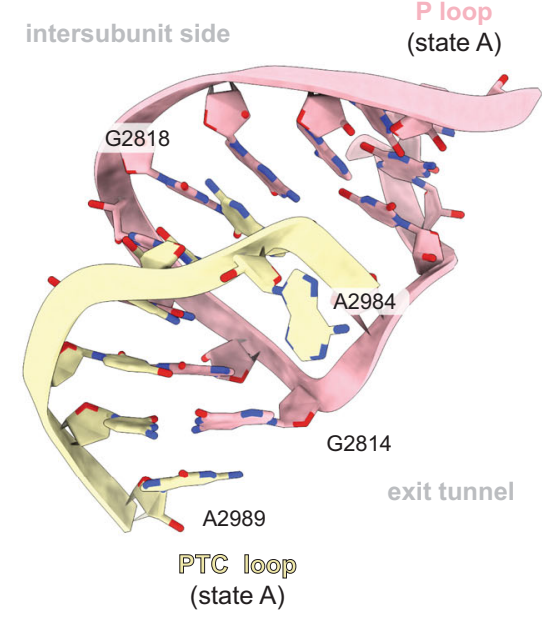

GAC

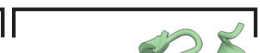

(1)
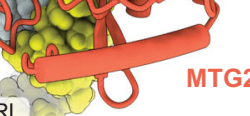

SRL

MTG2 G domain

d

intersubunit side

b
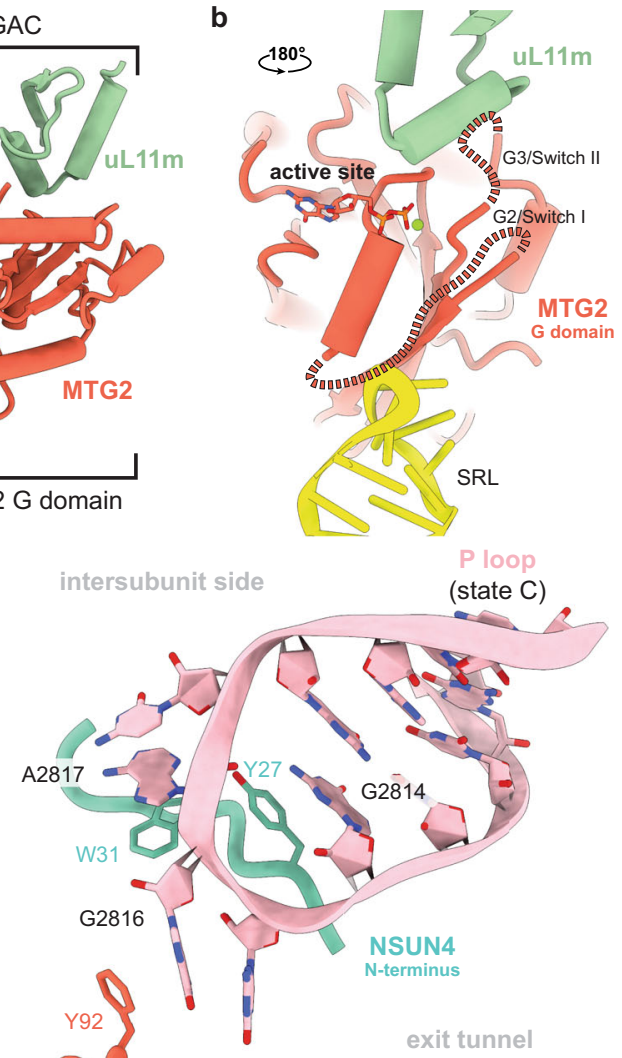

exit tunnel

MTG2
Obg domai

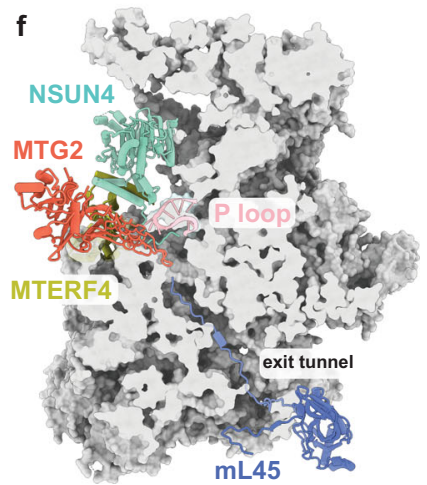

Fig. 3 Mitochondrial GTPase MTG2 interacts with the functional regions of the immature human mitoribosomal large subunit. a Interaction of the MTG2 (red cartoon) with the 16S rRNA and ribosomal proteins in state C. The N-terminal Obg domain of MTG2 contacts the peptidyl transferase center (PTC) region of the 16S rRNA (light cyan, PTC helices depicted individually, P loop in pink), whereas the G domain associates with the ribosomal GTPaseassociated center (GAC) components $\mathrm{uL} 11 \mathrm{~m}$ (green) and the sarcin-ricin loop (SRL). $\mathbf{b}$ Detailed view of $\mathrm{G}$ domain interactions with the GAC rotated by $180^{\circ}$ relative to panel (a). The Switch loops I and II are schematically indicated as dashed lines. The color key is the same as in panel (a). c The $16 \mathrm{~S}$ rRNA PTC loop (light yellow) stacking with the P loop (pink) as a result of an immature rRNA arrangement in state A. $\mathbf{d}$ Specific interactions of the NSUN4 Nterminal tail (teal) and the MTG2 Obg domain (red) with the P loop (pink). Amino acid residues involved in coordinating the P loop are highlighted. $\mathbf{e}$ Effects of N-terminal deletion of residues 26-36 in NSUN4 on its association with mitoribosomal fractions and 55S monosome formation were determined by immunoblotting. A continuous 10-30\% sucrose gradient was used to separate mitochondrial lysates from HEK 293T cells expressing the MTERF4 and NSUN4 mutant to determine their distribution and co-migration with mitochondrial ribosomal fractions. The small and large ribosomal subunit and polysomes in mitochondria isolated from NSUN4 $\Delta \mathrm{N}$ 26-36 transfected cells were followed by immunoblotting for mitochondrial ribosomal protein markers of the small (bS16m) and large (bL12m) ribosomal subunits. The input mitochondrial lysate was used as a positive control. The blots are representative of results obtained with at least three independent biological experiments. Source data are provided as a Source Data file. $\mathbf{f}$ Cross-section of the mitochondrial large subunit state C assembly intermediate. The spatial arrangement of assembly factors NSUN4 (teal), MTERF4 (light green), and MTG2 (red) ensure probing of the P loop (pink) and the entrance to the nascent polypeptide tunnel in the mitochondrial large subunit assembly intermediate (gray). The $\mathrm{mL} 45$ (blue) N-terminal tail occupies the exit tunnel, contributing to an inactive state of the subunit. 


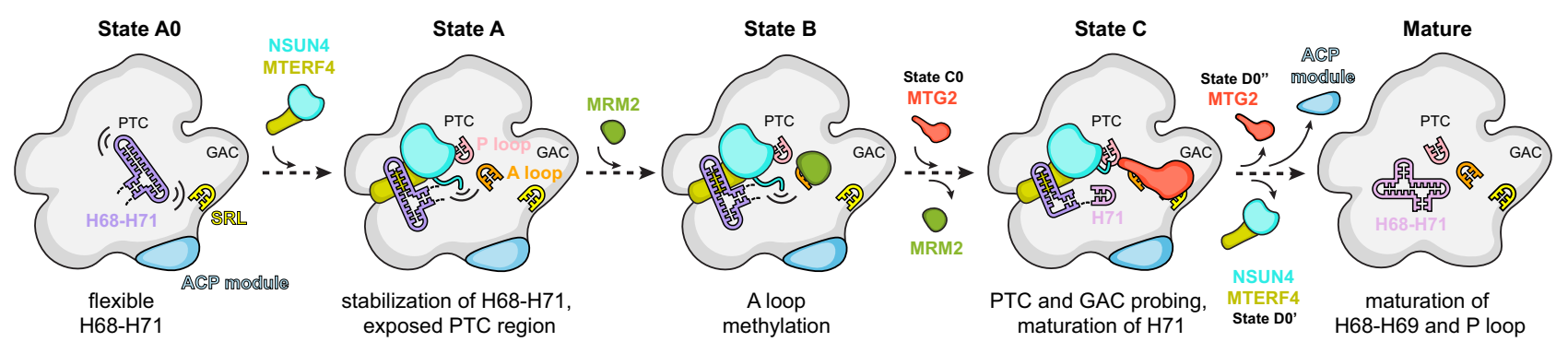

Fig. 4 Model for stepwise maturation of the human mitoribosomal large subunit aided by assembly factors. Eight classes, corresponding to distinct assembly states in this study, allow us to propose the order of events in the late stages of the human mitoribosomal large subunit assembly. Sequestering of the H68-H71 16S rRNA by assembly factors NSUN4 and MTERF4 exposes the functionally important regions of the large subunit that allows MRM2 to modify its target nucleotide in the A loop. Dissociation of MRM2 is followed by association of MTG2, a mitochondrial GTPase that performs a final quality check of the peptidyl transferase center (PTC) region as well as the sarcin-ricin loop (SRL) in the GTPase-associated center (GAC). Dissociation of all assembly factors, including MALSU1-LOR8F8-mt-ACP (ACP) module, results in the completion of the rRNA maturation and formation of a translationally competent particle.

ribosomes are likely to involve an equally diverse set of assembly factors across different species. Furthermore, while this work was in preparation, several manuscripts describing complementary results on the topic of mt-LSU maturation were published or uploaded in pre-print repositories ${ }^{32-35}$. Taken together, these results now provide a comprehensive description of the late stages of mitoribosomal large subunit assembly in human mitochondria. Furthermore, these results will help us understand the structural basis of mutations in the mitochondrial translational apparatus that are associated with neurodegenerative diseases ${ }^{36}$ and diverse progressive and fatal genetic disorders ${ }^{37,38}$.

\section{Methods}

Transient expression of MTG1-3xFLAG in HEK 293 EBNA cells. HEK 293 EBNA embryonic kidney cells, adapted to suspension growth in serum-free Ex-Cell medium, were obtained from the protein production and structure core facility at EPFL. The cell line was cultured at $37^{\circ} \mathrm{C}$ under $4.5 \% \mathrm{CO}_{2}$ in EX-CELL 293 serumfree medium for HEK 293 cells (Sigma), supplemented with $4 \mathrm{mM}$ L-glutamine. The cells were not tested for mycoplasma contamination.

The pcDNA3.1(+) plasmid encoding the C-terminally 3xFLAG-tagged MTG1 (UniProt ID Q9BT17) was ordered from GenScript. The inserted sequence was verified using a CMV forward primer at Microsynth. Cells with a concentration of $10^{6}$ cells $/ \mathrm{mL}$ were transfected with $1.5 \mathrm{mg}$ of DNA per liter of the culture using transfection reagent $40 \mathrm{kDa}$ PEI MAX (Polysciences, Inc.) in a 1:2 ratio. Cells were harvested and mitochondria were isolated $72 \mathrm{~h}$ post-transfection.

Isolation of mitochondria from HEK 293 EBNA cells. Mitochondria were isolated as previously described ${ }^{39,40}$ with few modifications. Briefly, the cell pellets were resuspended in ice-cold RSB hypo buffer $(10 \mathrm{mM}$ Tris- $\mathrm{HCl} \mathrm{pH} 7.5,10 \mathrm{mM}$ $\mathrm{NaCl}$, and $1.5 \mathrm{mM} \mathrm{MgCl}_{2}$ ) and allowed to swell for $10 \mathrm{~min}$. The swollen cells were opened with several strokes of a dounce homogenizer followed by immediate addition of $2.5 \times$ MS buffer (12.5 mM Tris- $\mathrm{HCl} \mathrm{pH} \mathrm{7.5,} 525 \mathrm{mM}$ mannitol, $175 \mathrm{mM}$ sucrose, $2.5 \mathrm{mM}$ EDTA, and $2.5 \mathrm{mM}$ DTT) to a final concentration of $1 \times$ MS buffer (5 mM Tris- $\mathrm{HCl} \mathrm{pH} 7.5,210 \mathrm{mM}$ mannitol, $70 \mathrm{mM}$ sucrose, $1 \mathrm{mM}$ EDTA and $1 \mathrm{mM}$ DTT). The homogenate was clarified at $1300 \times g$ and $4^{\circ} \mathrm{C}$ for $10 \mathrm{~min}$, followed by another $10 \mathrm{~min}$ centrifugation at $7500 \times g$ and $4{ }^{\circ} \mathrm{C}$. Finally, the supernatant and the crude mitochondria fraction were separated after centrifugation at $9500 \times \mathrm{g}$ and $4{ }^{\circ} \mathrm{C}$ for $10 \mathrm{~min}$. The mitochondria pellet was resuspended in $20 \mathrm{mM}$ HEPES-KOH pH 7.6, $250 \mathrm{mM}$ sucrose, and $1 \mathrm{mM}$ EDTA and applied to a layered sucrose gradient, consisting of $15 \%, 23 \%, 32 \%$, and $60 \%(\mathrm{w} / \mathrm{v})$ sucrose solutions in $20 \mathrm{mM}$ HEPES-KOH pH 7.6 and $1 \mathrm{mM}$ EDTA. After $70 \mathrm{~min}$ ultracentrifugation at $60,000 \times g$ and $4{ }^{\circ} \mathrm{C}$ using SW 32 Ti rotor, mitochondria band between $32 \%$ and $60 \%$ $(\mathrm{w} / \mathrm{v})$ sucrose solution was carefully collected, flash-frozen in liquid nitrogen, and stored at $-80^{\circ} \mathrm{C}$ until use.

Preparation of MTG1-3xFLAG-tagged mitochondrial ribosomes. Upon thawing of the mitochondria, 1.5 volumes of lysis buffer (20 mM HEPES-KOH pH 7.6, $100 \mathrm{mM} \mathrm{KCl}, 20 \mathrm{mM} \mathrm{MgCl}_{2}, 1.6 \%$ Triton X-100, supplemented with $1 \times$ cOmplete EDTA-free protease inhibitor cocktail (Roche)) was added, and mitochondria were lysed using a dounce homogenizer. Membranes were further solubilized by stirring for $15 \mathrm{~min}$ at $4^{\circ} \mathrm{C}$. The lysate was clarified by centrifugation at $20,800 \times g$ for $15 \mathrm{~min}$ at $4{ }^{\circ} \mathrm{C}$. Next, the supernatant was incubated for $1.5-2 \mathrm{~h}$ at $4{ }^{\circ} \mathrm{C}$ with anti-FLAG M2 affinity gel (Sigma) while gently mixing. The anti-FLAG M2 affinity gel (Sigma) was pre-equilibrated with three sequential column volumes of $0.1 \mathrm{M}$ glycine $\mathrm{HCl}$
pH 3.5 and washed with 10 volumes of TBS and wash buffer $(20 \mathrm{mM}$ HEPES-KOH $\mathrm{pH} 7.6,100 \mathrm{mM} \mathrm{KCl}$, and $20 \mathrm{mM} \mathrm{MgCl}_{2}$ ). After collecting the flow-through, the beads were washed with 10 column volumes of wash buffer. Bound mitoribosomes were eluted 3 times using elution buffer (20 mM HEPES-KOH pH 7.6, $100 \mathrm{mM}$ $\mathrm{KCl}, 20 \mathrm{mM} \mathrm{MgCl}_{2}$, and $100-200 \mu \mathrm{g} / \mathrm{mL} 3 \times \mathrm{FLAG}$ peptide), each time preceded by a $10-15$ min incubation at $4{ }^{\circ} \mathrm{C}$ with gentle mixing. Eluted fractions were pooled and subjected to a $2.5 \mathrm{~h}$ ultracentrifugation at $135,500 \times g$ at $4{ }^{\circ} \mathrm{C}$ using a TLA-55 rotor (Beckman-Coulter). Finally, the mitoribosome pellet was resuspended in mito-resuspension buffer (20 mM HEPES-KOH pH 7.6, $100 \mathrm{mM} \mathrm{KCl,} 20 \mathrm{mM}$ $\mathrm{MgCl}_{2}$, and $1 \mathrm{mM}$ DTT), yielding mt-LSU at a concentration of $\sim 40 \mathrm{nM}$. Samples from the key steps of the purification were subjected to western blot analysis (anti$3 \times$ FLAG antibody A8592, Sigma) (Supplementary Fig. 1).

Expression of NSUN4 variants in HEK 293T cells. Expression cassettes for NSUN4 variants were synthesized from overlapping oligonucleotides and cloned into pTwist CMV (Twist Bioscience). All NSUN4 variants were expressed as fusions to a C-terminal HA tag, while MTERF4 was C-terminally FLAG-tagged.

Human embryonic kidney (HEK 293T) cells were cultured at $37^{\circ} \mathrm{C}$ in humidified $95 \%$ air with $5 \% \mathrm{CO}_{2}$ in Dulbecco's modified essential medium (DMEM) (Gibco, Life Technologies) containing glucose $(4.5 \mathrm{~g} / \mathrm{L})$, L-glutamine $(2 \mathrm{mM}), 1 \mathrm{mM}$ sodium pyruvate, $50 \mu \mathrm{g} / \mathrm{ml}$ uridine, and $10 \%(\mathrm{v} / \mathrm{v})$ fetal bovine serum (FBS). The cells were tested and shown to be free off mycoplasma contamination. HEK 293T cells were plated at $60 \%$ confluence in $15 \mathrm{~cm}$ plates and transfected with mammalian expression plasmids in OptiMEM media (Invitrogen). $158 \mathrm{ng} / \mathrm{cm}^{2}$ of NSUN4 and MTERF4 plasmid DNA, in equal ratios, were transfected using Fugene HD (Roche). Cell incubations were carried out for $72 \mathrm{~h}$ following transfection and mitochondria were isolated as described previously ${ }^{41}$.

Sucrose gradients of mitochondrial ribosomes to analyze NSUN4 mutants. Sucrose gradient fractionation was carried out on purified mitochondria as previously described ${ }^{41}$. Briefly, isolated mitochondria were separated on a $10-30 \%$ sucrose gradient and lysed in $260 \mathrm{mM}$ sucrose, $100 \mathrm{mM} \mathrm{KCl}, 20 \mathrm{mM} \mathrm{MgCl}_{2}, 10$ $\mathrm{mM}$ Tris- $\mathrm{HCl} \mathrm{pH} 7.5,2 \%$ digitonin, $40 \mathrm{U} / \mathrm{ml}$ RNase inhibitor, and $1 \times$ cOmplete protease inhibitor cocktail (Roche) for $20 \mathrm{~min}$. After centrifugation at $9200 \times \mathrm{g}$ for $45 \mathrm{~min}$ at $4{ }^{\circ} \mathrm{C}$, the clarified lysates were loaded on a continuous $10-30 \%$ sucrose gradient containing $100 \mathrm{mM} \mathrm{KCl}, 20 \mathrm{mM} \mathrm{MgCl}_{2}, 10 \mathrm{mM}$ Tris- $\mathrm{HCl} \mathrm{pH} 7.5$, and the aforementioned RNase and protease inhibitors, followed by centrifugation at $71,000 \times g$ in an Optima Beckman Coulter preparative ultracentrifuge. Fractions were collected, and one-third of each fraction was precipitated with $0.02 \%$ sodium deoxycholate and $12 \%$ trichloroacetic acid, washed twice with acetone, and resolved by SDS-PAGE. Representative markers of the small and large ribosomal subunits were detected by immunoblotting as described below.

Immunoblotting. Specific proteins were detected using rabbit monoclonal antibodies against MRPL12 (bL12m) (16394-1-AP), MRPS16 (bS16m) (16735-1-AP), and FLAG (Sigma, F7425); and mouse monoclonal antibodies against HA (Cell Signaling, 2367). All primary antibodies were diluted 1:1000 using the Odyssey blocking buffer (LI-COR). IR dye $800 \mathrm{CW}$ goat anti-rabbit IgG or IR Dye 680LT goat anti-mouse IgG (LI-COR) secondary antibodies (diluted 1:10,000) were used, and the immunoblots were visualized using the Odyssey Infrared Imaging System (LI-COR).

Cryo-EM sample preparation and data acquisition. Quantifoil R2/2 holey carbon copper grids (Quantifoil Micro Tool) were prepared by applying an additional thin layer of continuous carbon, followed by glow-discharging for $15 \mathrm{~s}$ at $15 \mathrm{~mA}$ using an easiGlow Discharge cleaning system (PELCO). For both datasets, $4 \mu \mathrm{l}$ of 
resuspended MTG1-3×FLAG-tagged mitoribosome sample was directly applied onto the grid mounted in the Vitrobot chamber (Thermo Fisher Scientific) and incubated for $1 \mathrm{~min}$. Excess of buffer was blotted away, and the grid was immediately plunge frozen in 1:2 ethane:propane (Carbagas) at liquid nitrogen temperature. The Vitrobot chamber was kept at $4{ }^{\circ} \mathrm{C}$ and $100 \%$ humidity during the whole procedure. For the second dataset, NP-40 detergent was added to a final concentration of $0.001 \%$ just before applying the MTG1-3×FLAG-tagged mitoribosome sample onto the grid.

Both datasets were collected on a Titan Krios cryo-transmission electron microscope (Thermo Fisher Scientific) operating at $300 \mathrm{kV}$. For the first dataset, the microscope was equipped with a Falcon IIIEC Direct Electron Detector (FEI) and the movies were collected in integrating mode with a pixel size of $1.087 \AA / \mathrm{pix}$, 30 frames, and a total dose of $60 \mathrm{e}^{-} / \AA^{2}$, with defocus varying from -3.0 to $-0.6 \mu \mathrm{m}$. The second dataset was collected on a microscope equipped with a $\mathrm{K} 3$ detector (Gatan), mounted to a GIF Quantum LS imaging filter operated with an energy filter slit width of $20 \mathrm{eV}$. The movies were collected in counting and superresolution mode, with 40 frames and a total dose of $60 \mathrm{e}^{-} / \AA^{2}$ at a physical pixel size of $1.06 \AA /$ pix $(0.53 \AA /$ pix in super-resolution) with defocus varying from -3.0 to $-0.6 \mu \mathrm{m}$. The collection of both datasets was automated with the EPU software (Thermo Fisher Scientific).

Cryo-EM data processing. Both datasets were collected and processed independently. Unless stated otherwise, all processing steps were performed using cryoSPARC $3.1^{42}$

Movies of the first dataset collected on the Falcon IIIEC Direct Electron Detector (FEI) were drift-corrected and dose-weighted using MotionCor2 $2^{43}$, and the corrected 15,441 micrographs were imported into cryoSPARC ${ }^{43}$. The CTF parameters for each micrograph were estimated using patch-based CTF estimation, and 100 randomly selected micrographs were used to pick initial particles using a Laplacian-of-Gaussian filter-based method. These particles were subjected to a $2 \mathrm{D}$ classification. Classes resembling the large subunit of the human mitoribosome were used as a reference for picking particles from the whole dataset. The resulting 2,214,072 particles were extracted at 5.6-fold binning and subjected to 2D classification. Good-looking classes were selected and used for an ab-initio reconstruction to create the initial $3 \mathrm{D}$ model. The obtained model was then used as an input in homogenous refinement with all 1,064,609 selected particles from the 2D classification. The resulting refined map was used to create a mask covering the intersubunit side of the large subunit in UCSF Chimera ${ }^{44}$. Aligned particles, together with the mask, were used in a $3 \mathrm{D}$ Variability analysis ${ }^{45}$ with eight modes to solve and the resolution filtered to $6 \AA$. The particles were then divided into 10 clusters using $3 \mathrm{D}$ variability display.

The second dataset was pre-processed during collection using cryoSPARC live ${ }^{42}$. The pre-processing included 2-fold binning, drift-correction, doseweighting, CTF estimation, and particle picking using a Laplacian-of-Gaussian filter-based method, resulting in 10,682 processed micrographs and 1,626,837 extracted particles that were exported into cryoSPARC. The particles were then subjected to $2 \mathrm{D}$ classification, and classes resembling the mitoribosomal large subunit were selected, resulting in 833,994 particles. All particles were used for abinitio reconstruction, and the resulting cryo-EM map was used as an input model in homogenous refinement. The previously created intersubunit mask was resampled onto the new map using UCSF Chimera ${ }^{44}$ and together with the aligned particles was used for $3 \mathrm{D}$ variability analysis ${ }^{45}$ with five modes to solve and the resolution filtered to $10 \AA$. The particles were then divided into 25 clusters using 3D variability display.

Overall, the processing of both datasets resulted in similarly looking 3D classes. The only difference was states $\mathrm{B}$ and $\mathrm{D}^{\prime \prime}$, which were present only in the first and the second dataset, respectively. The key classes found in both datasets were reextracted at full-size and refined again, this time with per-particle defocus estimation ${ }^{46}$. The local resolution was calculated using a locally windowed FSC method as described in ref. ${ }^{47}$

Model building and refinement. Published structures of the mature human mitoribosome ${ }^{30}$ (PDB 6ZM6) and of a late assembly intermediate containing the ACP module ${ }^{17}$ (PDB 5OOL) were used as initial models and docked into the cryoEM maps using UCSF Chimera ${ }^{44}$. Composite models were assembled in PyMOL Molecular Graphics System, Version 2.1.5 (Schrödinger, LLC), followed by manual rebuilding of the proteins and nucleic acids using $\operatorname{Coot}^{48}$. For interpretation of the additional density features representing the assembly factors, the crystal structures of the human NSUN4-MTERF4 dimer ${ }^{24}$ (PDB 4FZV) and MRM2 (PDB 2NYU) were fitted and readjusted. For MTG2, an initial model was obtained using the Phyre2 modeling server ${ }^{49}$ based on a crystal structure of the E. coli homolog $\operatorname{ObgE}^{50}$ (PDB 5M04). The N-terminal Obg and C-terminal G domains were docked individually and rebuilt. For fitting the GDP and SAM cofactors, superimposed high-resolution structures were used as a guide.

The models were real-space refined for five cycles using Phenix version 1.19.1 ${ }^{51}$, while applying side-chain rotamer and Ramachandran restraints. The remaining discrepancies between the models and maps were detected and corrected using real space difference density maps and the geometry validation tools implemented in $\operatorname{Coot}^{48}$. The final model geometry was validated using MolProbity ${ }^{52}$

(Supplementary Table 1). To evaluate the quality of the fit of the refined models to the EM maps, real-space correlation coefficients $\left(\mathrm{CC}_{\text {mask }}\right)$ as well as the model versus map FSCs at the $\mathrm{FSC}=0.5$ criteria were calculated. The resulting resolutions were close to those calculated from the map half-sets at the $\mathrm{FSC}=0.143$ criteria (Supplementary Fig. 3).

Figure preparation. Molecular graphics and analyses were performed with UCSF Chimera $^{44}$ and UCSF ChimeraX ${ }^{53}$, developed by the Resource for Biocomputing Visualization, and Informatics at the University of California, San Francisco, with support from National Institutes of Health P41-GM103311 and R01-GM129325 and the Office of Cyber Infrastructure and Computational Biology, National Institute of Allergy and Infectious Diseases. Detailed views of cryo-EM map densities were created using The PyMOL Molecular Graphics System, Version 2.1.5 Schrödinger, LLC.

Reporting summary. Further information on research design is available in the Nature Research Reporting Summary linked to this article.

\section{Data availability}

The data that support this study are available from the corresponding author upon reasonable request. The atomic coordinates were deposited in the RCSB Protein Data Bank (PDB) under accession numbers 7ODR (state A), 7ODS (state B), and 7ODT (state C). The cryo-EM maps were deposited in the Electron Microscopy Data Bank (EMDB) under accession numbers EMD-12845 (state A), EMD-12846 (state B), EMD-12847 (state C), EMD-12848 (state A0), EMD-12849 (state C0), EMD-12850 (state D0'), EMD-12851 (state $\mathrm{D}^{\prime \prime}$ ), and EMD-12852 (state D). Source data are provided with this article.

Received: 1 April 2021; Accepted: 7 May 2021;

Published online: 16 June 2021

\section{References}

1. Greber, B. J. et al. The complete structure of the $55 \mathrm{~S}$ mammalian mitochondrial ribosome. Science 348, 303-308 (2015).

2. Amunts, A., Brown, A., Toots, J., Scheres, S. H. W. \& Ramakrishnan, V. The structure of the human mitochondrial ribosome. Science 348, 95-98 (2015).

3. Bieri, P., Greber, B. J. \& Ban, N. High-resolution structures of mitochondrial ribosomes and their functional implications. Curr. Opin. Struct. Biol. 49, 44-53 (2018)

4. Ferrari, A., Del'Olio, S. \& Barrientos, A. The diseased mitoribosome. FEBS Lett. https://doi.org/10.1002/1873-3468.14024 (2020).

5. Konikkat, S. \& Woolford, J. L. Principles of 60 S ribosomal subunit assembly emerging from recent studies in yeast. Biochem. J. 474, 195-214 (2017).

6. Davis, J. H. et al. Modular assembly of the bacterial large ribosomal subunit Cell 167, 1610-1622e15 (2016).

7. Nikolay, R. et al. Snapshots of native pre-50S ribosomes reveal a biogenesis factor network and evolutionary specialization. Mol. Cell 81, 1200-1215e9 (2021).

8. Jaskolowski, M. et al. Structural insights into the mechanism of mitoribosomal large subunit biogenesis. Mol. Cell 79, 629-644e4 (2020).

9. Soufari, H. et al. Structure of the mature kinetoplastids mitoribosome and insights into its large subunit biogenesis. Proc. Natl Acad. Sci. USA 117, 29851-29861 (2020).

10. Tobiasson, V. et al. Interconnected assembly factors regulate the biogenesis of mitoribosomal large subunit. EMBO J. 40, e106292 (2021).

11. Zeng, R., Smith, E. \& Barrientos, A. Yeast mitoribosome large subunit assembly proceeds by hierarchical incorporation of protein clusters and modules on the inner membrane. Cell Metab. 27, 645-656e7 (2018).

12. Bogenhagen, D. F., Ostermeyer-Fay, A. G., Haley, J. D. \& Garcia-Diaz, M. Kinetics and mechanism of mammalian mitochondrial ribosome assembly. Cell Rep. 22, 1935-1944 (2018).

13. Karbstein, K. Quality control mechanisms during ribosome maturation. Trends Cell Biol. 23, 242-250 (2013).

14. Maiti, P., Lavdovskaia, E., Barrientos, A. \& Richter-Dennerlein, R. Role of GTPases in driving mitoribosome assembly. Trends Cell Biol. 1-14 (2021) https://doi.org/10.1016/j.tcb.2020.12.008.

15. Silva, D. D. et al. Mitochondrial ribosome assembly in health and disease. Cell Cycle 14, 2226-2250 (2015).

16. Kummer, E. \& Ban, N. Mechanisms and regulation of protein synthesis in mitochondria. Nat. Rev. Mol. Cell Biol. 22, 307-325 (2021).

17. Brown, A. et al. Structures of the human mitochondrial ribosome in native states of assembly. Nat. Struct. Mol. Biol. 24, 866-869 (2017).

18. Jomaa, A. et al. Functional domains of the 50S subunit mature late in the assembly process. Nucleic Acids Res. 42, 3419-3435 (2014). 
19. Seffouh, A. et al. Structural consequences of the interaction of RbgA with a 50S ribosomal subunit assembly intermediate. Nucleic Acids Res. 47, 10414-10425 (2019).

20. Kotani, T., Akabane, S., Takeyasu, K., Ueda, T. \& Takeuchi, N. Human Gproteins, ObgH1 and Mtg1, associate with the large mitochondrial ribosome subunit and are involved in translation and assembly of respiratory complexes. Nucleic Acids Res. 41, 3713-3722 (2013).

21. Kim, H.-J. J. \& Barrientos, A. MTG1 couples mitoribosome large subunit assembly with intersubunit bridge formation. Nucleic Acids Res. 46, 8435-8453 (2018).

22. Cámara, Y. et al. MTERF4 regulates translation by targeting the methyltransferase NSUN4 to the mammalian mitochondrial ribosome. Cell Metab. 13, 527-539 (2011).

23. Metodiev, M. D. et al. NSUN4 is a dual function mitochondrial protein required for both methylation of $12 \mathrm{~S}$ rRNA and coordination of mitoribosomal assembly. PLoS Genet. 10, 1-11 (2014).

24. Yakubovskaya, E. et al. Structure of the essential MTERF4:NSUN4 protein complex reveals how an MTERF protein collaborates to facilitate rRNA modification. Structure 20, 1940-1947 (2012).

25. Spåhr, H., Habermann, B., Gustafsson, C. M., Larsson, N.-G. G. \& Hallberg, B. M. Structure of the human MTERF4-NSUN4 protein complex that regulates mitochondrial ribosome biogenesis. Proc. Natl Acad. Sci. USA 109, 15253-15258 (2012).

26. Rorbach, J. et al. MRM2 and MRM3 are involved in biogenesis of the large subunit of the mitochondrial ribosome. Mol. Biol. Cell 25, 2542-2555 (2014).

27. Lee, K.-W. \& Bogenhagen, D. F. Assignment of 2'-O-methyltransferases to modification sites on the mammalian mitochondrial large subunit $16 \mathrm{~S}$ ribosomal RNA (rRNA). J. Biol. Chem. 289, 24936-24942 (2014).

28. Maiti, P., Antonicka, H., Gingras, A.-C. C., Shoubridge, E. A. \& Barrientos, A. Human GTPBP5 (MTG2) fuels mitoribosome large subunit maturation by facilitating 16S rRNA methylation. Nucleic Acids Res. 48, 7924-7943 (2020).

29. Kummer, E. et al. Unique features of mammalian mitochondrial translation initiation revealed by cryo-EM. Nature 560, 263-267 (2018).

30. Itoh, Y. et al. Mechanism of membrane-tethered mitochondrial protein synthesis. Science 371, 846-849 (2021).

31. Saurer, M. et al. Mitoribosomal small subunit biogenesis in trypanosomes involves an extensive assembly machinery. Science 365, 1144-1149 (2019).

32. Cipullo, M., Gesé, G. V., Khawaja, A., Hällberg, B. M. \& Rorbach, J. Structural basis for late maturation steps of the human mitoribosomal large subunit. Nat. Commun. (2021).

33. Cheng, J., Berninghausen, O. \& Beckmann, R. A distinct assembly pathway of the human 39S late pre-mitoribosome. Preprint at bioRxiv https://doi.org/ 10.1101/2021.03.17.435838 (2021).

34. Hillen, H. S. et al. Structural basis of GTPase-mediated mitochondrial ribosome biogenesis and recycling. Nat. Commun. (2021).

35. Chandrasekaran, V. et al. Visualising formation of the ribosomal active site in mitochondria. Preprint at bioRxiv https://doi.org/10.1101/2021.03.19.436169 (2021).

36. Sun, N., Youle, R. J. \& Finkel, T. The mitochondrial basis of aging. Mol. Cell 61, 654-666 (2016).

37. Pearce, S., Nezich, C. L. \& Spinazzola, A. Mitochondrial diseases: translation matters. Mol. Cell. Neurosci. 55, 1-12 (2013).

38. Boczonadi, V. \& Horvath, R. Mitochondria: impaired mitochondrial translation in human disease. Int. J. Biochem. Cell Biol. 48, 77-84 (2014).

39. Clayton, D. A. \& Shadel, G. S. Isolation of mitochondria from cells and tissues. Cold Spring Harb. Protoc. 2014, pdb.top074542 (2014).

40. Aibara, S., Andréll, J., Singh, V. \& Amunts, A. Rapid isolation of the mitoribosome from HEK cells. J. Vis. Exp. https://doi.org/10.3791/57877 (2018).

41. Lee, R. G. et al. Cardiolipin is required for membrane docking of mitochondrial ribosomes and protein synthesis. J. Cell Sci. 133, jcs240374 (2020).

42. Punjani, A., Rubinstein, J. L., Fleet, D. J. \& Brubaker, M. A. cryoSPARC: algorithms for rapid unsupervised cryo-EM structure determination. Nat. Methods 14, 290-296 (2017)

43. Zheng, S. Q. et al. MotionCor2: anisotropic correction of beam-induced motion for improved cryo-electron microscopy. Nat. Methods 14, 331-332 (2017).

44. Pettersen, E. F. et al. UCSF Chimera-a visualization system for exploratory research and analysis. J. Comput. Chem. 25, 1605-1612 (2004).

45. Punjani, A. \& Fleet, D. J. 3D variability analysis: resolving continuous flexibility and discrete heterogeneity from single particle cryo-EM. J. Struct. Biol. 213, 107702 (2021).

46. Zivanov, J., Nakane, T. \& Scheres, S. H. W. Estimation of high-order aberrations and anisotropic magnification from cryo-EM data sets in RELION-3.1. IUCrJ 7, 253-267 (2020).
47. Cardone, G., Heymann, J. B. \& Steven, A. C. One number does not fit all: mapping local variations in resolution in cryo-EM reconstructions. J. Struct. Biol. 184, 226-236 (2013).

48. Emsley, P., Lohkamp, B., Scott, W. G. \& Cowtan, K. Features and development of Coot. Acta Crystallogr. Sect. D 66, 486-501 (2010).

49. Kelley, L. A., Mezulis, S., Yates, C. M., Wass, M. N. \& Sternberg, M. J. E. E. The Phyre2 web portal for protein modeling, prediction and analysis. Nat. Protoc. 10, 845-858 (2015).

50. Gkekas, S. et al. Structural and biochemical analysis of Escherichia coli ObgE, a central regulator of bacterial persistence. J. Biol. Chem. 292, 5871-5883 (2017).

51. Afonine, P. V. et al. Real-space refinement in PHENIX for cryo-EM and crystallography. Acta Crystallogr. Sect. D 74, 531-544 (2018).

52. Williams, C. J. et al. MolProbity: more and better reference data for improved all-atom structure validation. Protein Sci. 27, 293-315 (2018).

53. Goddard, T. D. et al. UCSF ChimeraX: meeting modern challenges in visualization and analysis. Protein Sci. 27, 14-25 (2018).

\section{Acknowledgements}

Authors thank A. Jomaa, E. Kummer, and S. Mattei for helpful discussions and creating a great working atmosphere. T.L. thanks R. Jost for help with the sample preparation, A. Picenoni and E. Kummer for help with visualizing the sample using negative stain EM, and A. Ries for establishing the cell culture laboratory. All authors would like to thank the ETH Zürich Scientific Centre for optical and electron microscopy (ScopeM) for access to electron microscopy equipment. T.L. is supported by an EMBO long-term fellowship (1074-2019). O.R. and A.F. are supported by fellowships and grants from the National Health and Medical Research Council and the Australian Research Council. This work was supported by the Swiss National Science Foundation (SNSF) (grant 31003A_182341), the National Center of Excellence in RNA and Disease of the SNSF (project funding 182880), and in part by the Roessler Prize, Ernst Jung Prize, and Otto Naegeli Prize for Medical Research, as well as by ETH Research Grant ETH-40 16-2 to N.B.

\section{Author contributions}

N.B. and T.L. designed the project. T.L., T.S., and M.J. prepared the sample for cryo-EM T.S. and M.S. established the methods for transfection of HEK cells with affinity-tagged mitochondrial proteins. A.S. and T.L. collected the cryo-EM data. M.J. and T.L. processed the cryo-EM data. M.L. built and performed coordinate refinement of the atomic models. T.L. and M.J. interpreted the structures. O.R., R.L., and A.F. performed in vivo experiments in HEK cells. T.L., M.J., A.F., and N.B. drafted the initial manuscript, all authors contributed to the final version.

\section{Competing interests}

The authors declare no competing interests.

\section{Additional information}

Supplementary information The online version contains supplementary material available at https://doi.org/10.1038/s41467-021-23811-8.

Correspondence and requests for materials should be addressed to N.B.

Peer review information Nature Communications thanks Yaser Hashem and the other, anonymous, reviewer(s) for their contribution to the peer review of this work.

Reprints and permission information is available at http://www.nature.com/reprints

Publisher's note Springer Nature remains neutral with regard to jurisdictional claims in published maps and institutional affiliations.

Open Access This article is licensed under a Creative Commons Attribution 4.0 International License, which permits use, sharing, adaptation, distribution and reproduction in any medium or format, as long as you give appropriate credit to the original author(s) and the source, provide a link to the Creative Commons license, and indicate if changes were made. The images or other third party material in this article are included in the article's Creative Commons license, unless indicated otherwise in a credit line to the material. If material is not included in the article's Creative Commons license and your intended use is not permitted by statutory regulation or exceeds the permitted use, you will need to obtain permission directly from the copyright holder. To view a copy of this license, visit http://creativecommons.org/ licenses/by/4.0/.

(c) The Author(s) 2021 